\title{
Incidences and Risk Factors for Postoperative Non- Union after Posterior Lumbar Interbody Fusion with Closed-Box Titanium Spacers
}

\author{
Tsunehiko Konomi, Akimasa Yasuda, Kanehiro Fujiyoshi, Yoshiyuki Yato, Takashi Asazuma \\ Department of Orthopaedic Surgery, Murayama Medical Center, National Hospital Organization, Tokyo, Japan
}

\begin{abstract}
Study Design: A retrospective, single-center clinical study with follow-up of more than 24 months.
Purpose: To evaluate the union rates and relevant risk factors for non-union after posterior lumbar interbody fusion (PLIF) using porous-coated closed-box titanium spacers.

Overview of Literature: Although the use of a closed-box interbody spacer for PLIF could avoid potential complications associated with the harvesting of autologous bone, few studies have reported detailed follow-up of fusion progression and risk factors for nonunion in the early postoperative period.

Methods: PLIF using closed-box spacers without filling the autologous bone was performed in 78 (88 levels) consecutive patients. Surgical procedures included PLIF using traditional pedicle screw fixation (PLIF, n=37), PLIF using cortical bone trajectory screw fixation (CBT-PLIF, $n=30$ ), and transforaminal lumbar interbody fusion with traditional pedicle screw fixation (TLIF, $n=11$ ). Lateral dynamic radiography and computed tomography findings were investigated, and the relationship between the union status and variables that may be related to the risk of non-union was tested statistically.

Results: The overall bone union rates at 12 and 24 months were $68.0 \%$ and $88.5 \%$, respectively. Incidences of bone cyst formation, subsidence, and retropulsion of spacers were $33.3 \%, 47.4 \%$, and $14.1 \%$, respectively. Union rates at 24 months were $94.6 \%$ in PLIF, $80.0 \%$ in CBT-PLIF, and $90.9 \%$ in TLIF. Multivariate logistic regression analyses showed that at 12 months postoperatively, the risk factor for non-union was age $>75$ years ( $p=0.02)$. In contrast, no significant risk factor was observed at 24 months.

Conclusions: These findings demonstrated the efficacy of interbody closed-box spacers for PLIF without the need to fill the spacer with autologous bone. However, the risk of non-union should be considered in elderly patients, especially intra-operatively and during the early postoperative stage.
\end{abstract}

Keywords: Posterior lumbar interbody fusion; Union rate; Radiographic assessment; Closed-box titanium spacer

\section{Introduction}

Posterior lumbar interbody fusion (PLIF) is a standard surgical technique for treating degenerative diseases of the lumbar spine. The objective of PLIF is to simultaneously stabilize the lumbar spine in a balanced alignment, reestablish the intervertebral disc height, and mechanically decompress both, the dural sac and the nerve root [1-3].

Received Jan 20, 2019; Revised Apr 7, 2019; Accepted Apr 17, 2019

Corresponding author: Tsunehiko Konomi

Department of Orthopaedic Surgery, Murayama Medical Center, National Hospital Organization, 2-37-1 Gakuen, Musashimurayama, Tokyo, 208-0011, Japan

Tel: +81-42-561-1221, Fax: +81-42-561-0997, E-mail: konomitsunehiko@gmail.com 
For fusion of the anterior column; bone grafts; hydroxyapatite blocks; or cages made of different materials, such as titanium, carbon, or polyether ether ketone (PEEK), and in different shapes are used to provide mechanical stability and promote solid interbody fusion. In most cases, the bone graft material is harvested from the iliac crest; however, in 1\%-39\% of cases, this results in complications of infection, pain, and sensory dysfunction [4-6].

In order to avoid such complications, the use of closedbox interbody spacer is an option that could avoid the need for filling with autologous bone and can thus be widely accepted in clinical practice. Kroppenstedt et al. [7] performed radiographical assessment of implantation of one or two spacers in PLIF surgery and found that segmental stability at 8 years postoperatively was achieved in $95.5 \%$ of cases with a single spacer and in $95.8 \%$ with two spacers. Although this report investigates the longterm radiographical findings, detailed follow-up of fusion progression and the relationship between the union status and related variables affecting non-union were not considered. Further, to our knowledge, no study has compared the union rates of PLIF using closed-box spacers with various pedicle screw insertion techniques. This study aimed to reveal the union rates and relevant risk factors for non-union after various PLIF techniques using closedbox interbody spacers in the early postoperative period.

\section{Materials and Methods}

This study is a single-center, retrospective clinical study. Total 84 consecutive patients who underwent PLIF using closed-box titanium spacers from April 2013 to March 2015 were eligible for this study. Seventy-eight patients who could be followed up for at least 24 months were selected retrospectively. We collected demographic data regarding age, sex, underlying cause of surgery, surgical procedure, level, number of interbody fusions, estimated blood loss, operating time, and surgeon experience. A senior surgeon was defined as a spine surgeon with at least 10 years of surgical experience, while a junior surgeon was someone with a surgical experience of $\leq 10$ years.

In all the surgical procedures, after intervertebral dissection, grafts of minced local bone were inserted into the intervertebral space before inserting the spacers. In the PLIF procedure, two spacers were inserted bilaterally into the intervertebral space using traditional pedicle screw fixation. In transforaminal lumbar interbody fu- sion (TLIF), the intervertebral space was accessed via a unilateral transforaminal approach, and a single spacer was used and inserted obliquely using traditional pedicle screw fixation. In the PLIF using cortical bone trajectory screws (CBT-PLIF), pedicle screws were inserted via mediolateral- and caudorostral-directed paths, and two spacers were inserted into the intervertebral space bilaterally. In this study, rectangular-shaped titanium block spacers (ProSpace; B. Braun Aesculap, Tuttlingen, Germany) with porous titanium coating were used. The heart of this implant is a solid titanium alloy core (Ti6AI4V), and the core is mantled with proven pure titanium coating. The mean porosity of the coating is approximately $37 \%$, and the size is controlled between 50 and $200 \mu \mathrm{m}$. This coating could create a bioactive surface to maximize the contact area for bony ingrowth due to its balanced pore depth, porosity, and roughness. Only ingrowth of bone into the spacer surface was intended; therefore, in theory, filling of the autologous bone was not required [7].

Bone union was assessed using lateral dynamic radiography and computed tomography (CT) findings following surgical treatment. We also reviewed the occurrence of bone cysts, subsidence, and retropulsion of the spacers. A 64-line multi-slice CT scanner was used, and 1-mmthick axial helical slices were obtained using a CT scanner. These slices were then used for CT-multi-planar reconstruction (CT-MPR). The criteria for radiological assessment of bone union were as follows (Fig. 1): (1) More than $3^{\circ}$ of motion on flexion-extension in the radiograph was considered to indicate non-union. (2) Non-union was defined as the presence of a visible gap between the vertebral

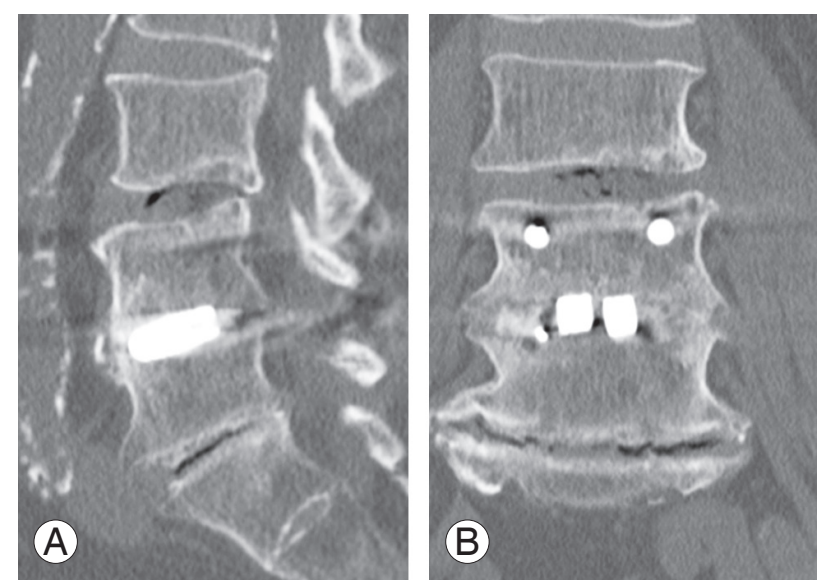

Fig. 1. (A, B) Bone union of interbody fusion is defined as the condition where no gap is visible between the endplates and the spacer, and/or the mobility on flexion-extension radiographs is $\leq 3^{\circ}$. 
endplate and spacer or radiolucency around the pedicle screws on a radiograph and/or a CT-MPR image [8-10]. A bone cyst was defined as cystic formation on and/or in an endplate [9]. Radiographic subsidence was defined as any compromise of either endplate [11]. Retropulsion of a spacer was defined as any movement of the posterior margin of the spacer in comparison with the lateral view radiograph taken immediately after surgical treatment. Intervertebral disc angle was calculated by the lateral view radiograph in the pre-, post-, and 24 months postoperative stage. All the patients wore a lumbosacral orthosis for at least 3 months postoperatively.

The modified MacNab criteria were used for assessing the clinical outcomes. It defined excellent outcome as no pain and no restriction of activity; good outcome as occasional pain, without the need for medication and no restriction of activity; fair outcome as somewhat improvement and need of medication, with some restriction of daily activity; and poor outcome as no improvement or worsening, and/or need for further operative intervention $[12,13]$.

The included patients were classified into two groups as per the condition of bone union at 24 months, and differences between the two groups were assessed using Fisher's exact test for categorical variables (age, sex, osteoporosis, surgical characteristics, surgical procedures, and CT findings) using Prism 5.0 (GraphPad Software Inc., La Jolla, CA, USA). In order to elucidate the prognostic risk factors for non-union at 12 and 24 months, multivariate logistic regression analyses were performed with EZR (Saitama Medical Center, Jichi Medical University, Saitama, Japan), a graphical user interface for R (The R Foundation for Statistical Computing, Vienna, Austria) [14]. More precisely, it is a modified version of $\mathrm{R}$ commander designed to add statistical functions frequently used in biostatistics. Odds ratios for non-union and their $95 \%$ confidence intervals were calculated as an approximation of the relative risk estimates. The factors included in the multivariate model were sex, two-level fusion and those factors with a nonunion rate at 24 months of at least $15 \%$ (Table 1): age $\geq 75$ years, junior surgeon, bone cyst, subsidence, retropulsion, and CBT-PLIF. A value of $p<0.05$ was considered statistically significant.

All procedures performed in studies involving human participants were in accordance with the ethical standards of the institutional and/or national research committee and with the 1964 Helsinki declaration and its later
Table 1. Rates of non-union at 24 months according to clinical factors

\begin{tabular}{lcc}
\hline Factors & No. of non-union (\%) & $p$-value \\
Gender (female) & $5(12.8)$ & 1.00 \\
Age (yr) & & \\
$\geq 70$ & $3(9.0)$ & 0.73 \\
$\geq 75$ & $3(18.8)$ & 0.38 \\
\hline$\geq 80$ & $1(14.0)$ & 1.00 \\
\hline Surgical characteristics & & \\
\hline Osteoporosis & $6(13.0)$ & 0.79 \\
\hline Junior surgeon & $8(14.6)$ & 0.27 \\
\hline Multilevel fusion & $1(10.0)$ & 1.00 \\
\hline Surgical procedure & & \\
\hline PLIF & $2(5.4)$ & 0.16 \\
\hline CBT-PLIF & $6(20.0)$ & 0.08 \\
\hline TLIF & $1(9.1)$ & 1.00 \\
\hline Computed tomography findings & & \\
\hline Bone cyst & $6(23.1)$ & 0.05 \\
\hline Subsidence & $6(16.2)$ & 0.30 \\
\hline Retropulsion & $3(27.3)$ & 0.11 \\
\hline PLF posterior lumbar interbody & & \\
\hline
\end{tabular}

PLIF, posterior lumbar interbody fusion; CBT-PLIF, PLIF using cortical bone trajectory screws; TLIF, transforaminal lumbar interbody fusion.

amendments or comparable ethical standards. Informed consent was obtained from all individual participants included in the study.

\section{Results}

The study population of 78 patients included 39 men and 39 women, with a mean age was 66.0 years (range, 26-82 years) (Table 2). The patients' diagnoses were as follows: lumbar spinal stenosis $(\mathrm{n}=47)$, degenerative spondylolisthesis $(n=16)$, lumbar disk herniation $(n=9)$, ischemic spondylolysis $(\mathrm{n}=5)$, and adjacent segmental disorder $(\mathrm{n}=1)$. Sixty-eight patients underwent single-level fusion, and 10 underwent two-level fusion. Of the total 88 disks, fusion segments were as follows: L2/3 ( $\mathrm{n}=1), \mathrm{L} 3 / 4(\mathrm{n}=10)$, L4/5 ( $n=53)$, and L5/S1 $(n=24)$. Surgical procedures consisted of conventional PLIF $(n=37)$, CBT-PLIF $(n=30)$, and TLIF $(\mathrm{n}=11)$. The mean operation time was 177.1 minutes, and the mean estimated blood loss was 218.5 $\mathrm{mL}$.

Total bone union rates at 12 and 24 months were $67.9 \%$ and $88.5 \%$, respectively (Tables 1,3 ). Bone union rates at 24 months according to each surgery-related variable were 94.5\% in PLIF, $80.0 \%$ in CBT-PLIF, and $90.9 \%$ in TLIF, 
Table 2. Patient demographics

\begin{tabular}{|c|c|}
\hline Characteristic & Value \\
\hline Age (yr) & $66.0 \pm 13.4$ \\
\hline Gender (male:female) & 39:39 \\
\hline \multicolumn{2}{|l|}{ Disease } \\
\hline Lumbar canal stenosis & $47(60)$ \\
\hline Degenerative spondylolisthesis & $16(21)$ \\
\hline Lumbar disc herniation & $9(12)$ \\
\hline Ischemic spondylolysis & $5(6)$ \\
\hline Adjacent segmental disorder & $1(1)$ \\
\hline Estimated blood loss (mL) & $218.5 \pm 209.9$ \\
\hline Operation time (min) & $177.1 \pm 70.4$ \\
\hline \multicolumn{2}{|l|}{ Surgeon } \\
\hline Junior surgeon & $55(70)$ \\
\hline Senior surgeon & $23(30)$ \\
\hline \multicolumn{2}{|l|}{ No. of fusion } \\
\hline Single level fusion & $68(87)$ \\
\hline Two-level fusion & $10(13)$ \\
\hline \multicolumn{2}{|l|}{ Level } \\
\hline $\mathrm{L} 2 / 3$ & $1(1)$ \\
\hline $\mathrm{L} 3 / 4$ & $10(12)$ \\
\hline$\lfloor 4 / 5$ & $53(60)$ \\
\hline L5/S1 & $24(27)$ \\
\hline \multicolumn{2}{|l|}{ Surgical procedure } \\
\hline PLIF & $37(47)$ \\
\hline CBT-PLIF & $30(39)$ \\
\hline TLIF & $11(14)$ \\
\hline
\end{tabular}

Values are presented as mean \pm standard deviation or number (\%). PLIF, posterior lumbar interbody fusion; CBT-PLIF, PLIF using cortical bone trajectory screws; TLIF, transforaminal lumbar interbody fusion.

with bone union achieved in $95.7 \%$ of the patients operated on by senior surgeons and $85.5 \%$ of those operated by junior surgeons. A bone cyst was detected in 26 cases (33.3\%), and subsidence of the spacer was detected in 37 cases (47.4\%). Retropulsion of the spacer was detected in 11 patients (14.1\%); one of these patients experienced severe radiculopathy and required revision surgery. For the PLIF group, 13 patients (35.1\%) had excellent results, 18 patients (48.7\%) achieved good results, five patients (13.5\%) had fair results, and one patient $(2.7 \%)$ had poor result. In the CBT-PLIF group, eight patients (26.7\%) had excellent results, 15 (50.0\%) had good results, five patients (16.7\%) had fair results, and two patients (6.7\%) had poor results. In the TLIF group, six patients (54.6\%) had excel- lent results, four patients (36.4\%) had good results, and one patient (9.1\%) had a fair result (Table 3).

Intervertebral disc angles before, after, and 24 months after the surgery were $3.8^{\circ}, 8.1^{\circ}$, and $6.3^{\circ}$ in the union group and $4.1^{\circ}, 8.3^{\circ}$, and $5.4^{\circ}$ in the non-union group, respectively, without any statistical difference. To evaluate the effect on non-union, variables were compared between the union and non-union groups (Table 1). Univariate analyses revealed that no variable had a significant effect on non-union at 24 months. Thus, multivariate regression analyses were performed using all the variables with non-union rates of $>15 \%$ at 24 months, sex, and twolevel fusion. In the result, age $>75$ years was the only risk factor for non-union at 12 months $(p=0.02)$. In contrast, no variable was identified as a relevant significant risk factor of non-union at 24 months (Tables 4,5 ).

\section{Discussion}

In this study, we addressed the incidence and risk factor for non-union of closed-box titanium spacers without filling with autologous bone following various PLIF procedures. Multivariate logistic regression analyses revealed that the only risk factor for non-union at 12 months was age $>75$ years, while there was no significant risk factor at 24 months after the surgery.

Several studies have reported interbody bone union rates after PLIF surgery with autologous bone or other types of cage filling with autologous bone of $82 \%-90 \%$ in PEEK cages $[9,15,16], 96 \%$ in titanium cages [10], $87 \%-94 \%$ in carbon fiber reinforced polymer cages [8,17], $88 \%-96 \%$ in PEEK cages coated with carbon fiber [18], and $96 \%$ in hydroxyapatite blocks with autogenous iliac bone [19]. In this study, the overall bone union rate at 24 months was $88.5 \%$, similar to previous reports, indicating fusion potential of closed-box titanium spacers as an intervertebral supporter following PLIF. Although there was no difference in the union rates among three surgical procedures from the statistical standpoint, the union rate of CBT-PLIF was lower (56.7\% at 12 months and $80.0 \%$ at 24 months) than that of others $(75.6 \%$ and $94.5 \%$ in PLIF and $72.7 \%$ and $90.9 \%$ in TLIF at 12 and 24 months, respectively). Sakaura et al. [18] reported that the union rate of CBT-PLIF was lower than that of conventional PLIF and speculated that shorter lever arm in the screw trajectory might result in weakness of impaction between cages and vertebral bodies in the perpendicular direc- 
Table 3. Rates of bone union, CT findings, and clinical outcomes according to surgical procedures

\begin{tabular}{|c|c|c|c|c|}
\hline Variable & PLIF (n=37) & CBT-PLIF (n=30) & TLIF $(n=11)$ & Total $(\mathrm{n}=78)$ \\
\hline \multicolumn{5}{|l|}{ CT findings } \\
\hline Bone cyst & $12(32.4)$ & $11(36.6)$ & $3(27.3)$ & $26(33.3)$ \\
\hline Subsidence & $15(40.5)$ & $15(50.0)$ & $7(63.6)$ & $37(47.4)$ \\
\hline Retropulsion & $3(8.1)$ & $8(26.7)$ & $0(0.0)$ & $11(14.1)$ \\
\hline Bone union (12 mo) & $28(75.6)$ & $17(56.7)$ & $8(72.7)$ & $53(68.0)$ \\
\hline Bone union (24 mo) & $35(94.6)$ & $24(80.0)$ & 10 (90.9) & 69 (88.5) \\
\hline \multicolumn{5}{|c|}{ Clinical outcomes at 24 mo (modified MacNab criteria) } \\
\hline Excellent & $13(35.1)$ & $8(26.7)$ & $6(54.6)$ & $27(34.6)$ \\
\hline Good & $18(48.7)$ & $15(50.0)$ & $4(36.4)$ & $37(47.4)$ \\
\hline Fair & $5(13.5)$ & $5(16.7)$ & $1(9.1)$ & $11(14.1)$ \\
\hline Poor & $1(2.7)$ & $2(6.7)$ & $0(0)$ & $3(3.9)$ \\
\hline
\end{tabular}

Values are presented as number (\%).

CT, computed tomography; PLIF, posterior lumbar interbody fusion; CBT-PLIF, PLIF using cortical bone trajectory screws; TLIF, transforaminal lumbar interbody fusion.

Table 4. Prognostic factors for the occurrence of non-union at 12 months

\begin{tabular}{lcc}
$\begin{array}{l}\text { Explanatory } \\
\text { variable }\end{array}$ & $\begin{array}{c}\text { Odds ratio } \\
\text { confidence interval) }\end{array}$ & $p$-value \\
Gender & $0.85(0.27-2.67)$ & 0.78 \\
\hline Age $\geq 75 \mathrm{yr}$ & $4.67(1.18-18.40)$ & 0.02 \\
\hline Junior surgeon & $0.54(0.14-2.06)$ & 0.37 \\
\hline Multilevel fusion & $1.95(0.36-10.60)$ & 0.44 \\
\hline Bone cyst & $2.84(0.89-9.06)$ & 0.08 \\
\hline Subsidence & $0.93(0.29-2.99)$ & 0.91 \\
\hline Retropulsion & $3.76(0.74-19.10)$ & 0.11 \\
CBT-PLIF & $1.54(0.42-5.57)$ & 0.51 \\
\hline
\end{tabular}

CBT-PLIF, posterior lumbar interbody fusion using cortical bone trajectory screws.

tion. Retropulsion of cages is also believed to be caused by insufficient compressive force between the cages and vertebral bodies $[20,21]$. Although the incidence of retropulsion in CBT-PLIF was highest among the three surgical procedures, it did not affect the union condition significantly. Matsukawa et al. [22] experimentally demonstrated that the screw diameter and length within the vertebral body are important factors in axial compression loading, suggesting that longer screw insertion could overcome the weakness of CBT screw techniques.

A positive cyst formation in the endplate is strongly correlated with non-union or delayed-union following PLIF [9]. Subsidence is also demonstrated to be related to non-union, with the reported incidence of subsidence
Table 5. Prognostic factors for the occurrence of non-union at 24 months

\begin{tabular}{lcc} 
Explanatory variable & $\begin{array}{c}\text { Odds ratio }(95 \% \\
\text { confidence interval) }\end{array}$ & $p$-value \\
\hline Gender & $0.91(0.16-5.17)$ & 0.92 \\
\hline Age $\geq 75 \mathrm{yr}$ & $2.47(0.37-16.40)$ & 0.35 \\
\hline Junior surgeon & $8.33(0.69-101.00)$ & 0.10 \\
\hline Multilevel fusion & $0.92(0.05-16.10)$ & 0.95 \\
\hline Bone cyst & $4.09(0.76-22.10)$ & 0.10 \\
\hline Subsidence & $1.57(0.27-9.05)$ & 0.61 \\
\hline Retropulsion & $1.86(0.25-14.10)$ & 0.55 \\
\hline CBT-PLIF & $4.98(0.78-31.70)$ & 0.09 \\
\hline
\end{tabular}

CBT-PLIF, posterior lumbar interbody fusion using cortical bone trajectory screws.

being about $8 \%-17 \%$ in PEEK cages [11]. In this study, although no significant difference was observed between union and non-union in cases with bone cyst or subsidence, the incidences were higher than those in previous reports. We hypothesize that there are three reasons for higher incidences, including the hardness of closed-box titanium spacers, the fragility of the spinal bone in elderly patients, and/or traumatic intra-operative procedures. In fact, Young's modulus of titanium alloy (Ti6AI4V) is about $110 \mathrm{GPa}$ [23]. Simultaneously, metallic biomaterial requires a low Young's modulus close to that of the bone (10-30 GPa) to prevent born resorption [24]. Although there was no relationship between the presence of osteoporosis and non-union, older age ( $>75$ years) was the only 
statistically significant risk factor for non-union at 12 months. Further, the union rate of junior surgeons tends to be lower than that of senior surgeons. These facts may cause insidious disruption of the intervertebral endplate in elderly patients during traumatic intra-operative procedures and/or use of hard closed-box titanium spacers, resulting in a higher incidence of subsidence and cyst formation. In sum, in any case, the insertion of spacers into the intervertebral space should be carefully performed in a gentle and atraumatic manner.

The limitations of this study were its retrospective design, the relatively small number of patients assessed, and the fact that the methods of surgical treatment differed as per the clinician who managed the patients. Although the number of patients was small and the methods of management were different from a statistical standpoint, our findings demonstrated stable clinical results in PLIF using closedbox spacers without filling the autologous bone and a low complication rate related to revision surgery. In fact, closed-box titanium spacers had no competitive advantage over other types of cages with respect to bone union, especially in cases with CBT-PLIF. Thus, to our knowledge, this was the first study to reveal risk factors for non-union during the early postoperative stage after PLIF with the use of spacers and to compare fusion progression from different viewpoints. Almost all patients achieved clinically satisfactory outcomes. However, patient-reported outcome measures were not assessed; therefore, further studies that involve a randomized prospective trial design and aim to evaluate the efficacy of closed-box spacers from multiple viewpoints is necessary.

\section{Conclusions}

This study demonstrated bone union rates and risk factors for non-union after alternative PLIF procedures using solid closed-box titanium spacers without filling the autologous bone. Although most patients showed a stable union rate, older age was associated with the risk of non-union at 12 months postoperatively. Therefore, when performing PLIF with spacers, surgeons should possess skillful surgical techniques and take care to prevent non-union, especially intra-operatively and during the early postoperative stage.

\section{Conflict of Interest}

No potential conflict of interest relevant to this article was reported.

\section{Acknowledgments}

We gratefully acknowledge Dr Yoshihide Yanai and Dr Hideaki Ohne, who assisted this study.

\section{ORCID}

Tsunehiko Konomi: https://orcid.org/0000-0001-6704-7573

\section{References}

1. Jaslow IA. Intercorporal bone graft in spinal fusion after disc removal. Surg Gynecol Obstet 1946;82:2158.

2. Cloward RB. Posterior lumbar interbody fusion updated. Clin Orthop Relat Res 1985;(193):16-9.

3. Cloward RB. The treatment of ruptured lumbar intervertebral discs; criteria for spinal fusion. Am J Surg 1953;86:145-51.

4. Keller EE, Triplett WW. Iliac bone grafting: review of 160 consecutive cases. J Oral Maxillofac Surg 1987;45:11-4.

5. Summers BN, Eisenstein SM. Donor site pain from the ilium: a complication of lumbar spine fusion. J Bone Joint Surg Br 1989;71:677-80.

6. Younger EM, Chapman MW. Morbidity at bone graft donor sites. J Orthop Trauma 1989;3:192-5.

7. Kroppenstedt S, Gulde M, Schonmayr R. Radiological comparison of instrumented posterior lumbar interbody fusion with one or two closed-box plasmapore coated titanium cages: follow-up study over more than seven years. Spine (Phila Pa 1976) 2008;33:2083-8.

8. Ito Z, Matsuyama Y, Sakai Y, et al. Bone union rate with autologous iliac bone versus local bone graft in posterior lumbar interbody fusion. Spine (Phila $\mathrm{Pa}$ 1976) 2010;35:E1101-5.

9. Fujibayashi S, Takemoto M, Izeki M, Takahashi Y, Nakayama T, Neo M. Does the formation of vertebral endplate cysts predict nonunion after lumbar interbody fusion? Spine (Phila Pa 1976) 2012;37:E1197202. 
10. Lee JH, Jeon DW, Lee SJ, Chang BS, Lee CK. Fusion rates and subsidence of morselized local bone grafted in titanium cages in posterior lumbar interbody fusion using quantitative three-dimensional computed tomography scans. Spine (Phila Pa 1976) 2010;35:1460-5.

11. Malham GM, Parker RM, Blecher CM, Seex KA. Assessment and classification of subsidence after lateral interbody fusion using serial computed tomography. J Neurosurg Spine 2015;23:589-97.

12. Macnab I. Negative disc exploration: an analysis of the causes of nerve-root involvement in sixty-eight patients. J Bone Joint Surg Am 1971;53:891-903.

13. Sinkemani A, Hong X, Gao ZX, et al. Outcomes of microendoscopic discectomy and percutaneous transforaminal endoscopic discectomy for the treatment of lumbar disc herniation: a comparative retrospective study. Asian Spine J 2015;9:833-40.

14. Kanda Y. Investigation of the freely available easy-touse software 'EZR' for medical statistics. Bone Marrow Transplant 2013;48:452-8.

15. Lee JH, Lee JH, Park JW, Lee HS. Fusion rates of a morselized local bone graft in polyetheretherketone cages in posterior lumbar interbody fusion by quantitative analysis using consecutive three-dimensional computed tomography scans. Spine J 2011;11:647-53.

16. Nakashima H, Yukawa Y, Ito K, et al. Extension CT scan: its suitability for assessing fusion after posterior lumbar interbody fusion. Eur Spine J 2011;20:1496502.

17. Fogel GR, Toohey JS, Neidre A, Brantigan JW. Fusion assessment of posterior lumbar interbody fu- sion using radiolucent cages: X-ray films and helical computed tomography scans compared with surgical exploration of fusion. Spine J 2008;8:570-7.

18. Sakaura H, Miwa T, Yamashita T, Kuroda Y, Ohwada T. Posterior lumbar interbody fusion with cortical bone trajectory screw fixation versus posterior lumbar interbody fusion using traditional pedicle screw fixation for degenerative lumbar spondylolisthesis: a comparative study. J Neurosurg Spine 2016;25:591-5.

19. Asazuma T, Masuoka K, Motosuneya T, Tsuji T, Yasuoka H, Fujikawa K. Posterior lumbar interbody fusion using dense hydroxyapatite blocks and autogenous iliac bone: clinical and radiographic examinations. J Spinal Disord Tech 2005;18 Suppl:S41-7.

20. Lee DY, Park YJ, Song SY, Jeong ST, Kim DH. Risk factors for posterior cage migration after lumbar interbody fusion surgery. Asian Spine J 2018;12:59-68.

21. Kimura H, Shikata J, Odate S, Soeda T, Yamamura S. Risk factors for cage retropulsion after posterior lumbar interbody fusion: analysis of 1070 cases. Spine (Phila Pa 1976) 2012;37:1164-9.

22. Matsukawa K, Yato Y, Imabayashi H, et al. Biomechanical evaluation of fixation strength among different sizes of pedicle screws using the cortical bone trajectory: what is the ideal screw size for optimal fixation? Acta Neurochir (Wien) 2016;158:465-71.

23. Rho JY, Tsui TY, Pharr GM. Elastic properties of human cortical and trabecular lamellar bone measured by nanoindentation. Biomaterials 1997;18:1325-30.

24. Niinomi M, Liu Y, Nakai M, Liu H, Li H. Biomedical titanium alloys with Young's moduli close to that of cortical bone. Regen Biomater 2016;3:173-85. 